En la Secretaría de Teoría de la Educación. Revista Interuniversitaria se recibieron durante 2018 un total de 94 trabajos pertenecientes a 124 autores, con procedencia de instituciones científicas extranjeras en el treinta por ciento de los casos. De los 94 trabajos recibidos, 24 han sido publicados y 70 han sido rechazados. La tasa de rechazo resultante es del $75 \%$.

El proceso de evaluación de los trabajos ha sido doblemente anónimo, con participación de 43 revisores externos que emitieron un total de 150 informes. El $30 \%$ de los revisores pertenece a instituciones científicas extranjeras.

Albert Gómez, María José

Almeida de, Rogério

Álvarez Castillo, José Luis

Argos González, Javier

Bernal Guerrero, Antonio

Dacosta Martínez, Arsenio F.

Fernández-Salinero, Carolina

Fuentes Gómez-Calcerrada, Juan Luis

Gárate Rivera, Alberto

Gil Cantero, Fernando

Gozálvez Pérez, Vicent

Jordán Sierra, José Antonio

King, Chris

Leal Filho, Walter

Lee, Elsa

Linares Borboa, Luis Enrique

Madrussan, Elena

Martín Solbes, Víctor M.

Mélich i Sangrá, Joan Carles

Mínguez Vallejos, Ramón

Montanero Fernández, Manuel

Navarrete Salvador, Antonio

Pedros, Maria Arminda

Peña Calvo, José Vicente

Pessoa Rivero, Teresa

Prats Gil, Enric
Universidad Nacional de

Educación a Distancia

Universidade de São Paulo, Brasil

Universidad de Córdoba

Universidad de Cantabria

Universidad de Sevilla

Universidad de Salamanca

Universidad Complutense de Madrid

Universidad Complutense de Madrid

Cetyx Universidad, México

Universidad Complutense de Madrid

Universidad de Valencia

Universidad de Barcelona

Keele University, United Kingdom

Hamburg University of Applied

Sciences, Germany

University of Cambridge, United Kingdom

CETYX Universidad, México

Università di Torino, Italia

Universidad de Málaga

Universidad de Barcelona

Universidad de Murcia

Universidad de Extremadura

Universidad de Cádiz

Universidad de Coímbra, Portugal

Universidad de Oviedo

Universidad de Coímbra, Portugal

Universidad de Barcelona 
Rodríguez Neira, Teófilo

Romero Pérez, Clara

Romero Sánchez, Eduardo

Ruiz Corbella, Marta

Sa, Patricia

Sánchez Carracedo, Fermín

Santos Gómez, Marcos

Santos Rego, Miguel Ángel

Sanz Ponce, Roberto

Salonen, Arto

Saura Casanova, Geo

Soromenho-Marques, Viriato

Touriñán López, José Manuel

Vázquez Verdera, Victoria

Vega Mancera, Felipe

Vera Vila, Julio
Universidad de Oviedo

Universidad de Sevilla

Universidad de Murcia

Universidad Nacional de

Educación a Distancia

Universidad de Aveiro, Portugal

Universidad Politécnica de Cataluña

Universidad de Granada

Universidad de Santiago de Compostela

Universidad de Valencia

Helsinki University of Applied

Sciences, Finland

Universidad Autónoma de Barcelona

Universidad de Lisboa, Portugal

Universidad de Santiago de Compostela

Universidad de Valencia

Universidad de Málaga

Universidad de Málaga 
ISSN 1130-3743

\section{DATOS DEL ARTíCUlo}

Título:

Fecha de recepción:

Fecha de devolución:

Evaluador:
Fecha de envío a evaluación:

Fecha de aceptación definitiva:

\section{Criterios De Evaluación}

1. Adecuación del título al contenido del trabajo

2. Estructura y contenido del resumen y palabras clave

$\begin{array}{llllllllll}1 & 2 & 3 & 4 & 5 & 6 & 7 & 8 & 9 & 10 \\ 1 & 2 & 3 & 4 & 5 & 6 & 7 & 8 & 9 & 10 \\ 1 & 2 & 3 & 4 & 5 & 6 & 7 & 8 & 9 & 10 \\ 1 & 2 & 3 & 4 & 5 & 6 & 7 & 8 & 9 & 10 \\ 1 & 2 & 3 & 4 & 5 & 6 & 7 & 8 & 9 & 10 \\ 1 & 2 & 3 & 4 & 5 & 6 & 7 & 8 & 9 & 10 \\ 1 & 2 & 3 & 4 & 5 & 6 & 7 & 8 & 9 & 10 \\ & & & & & & & & & \\ 1 & 2 & 3 & 4 & 5 & 6 & 7 & 8 & 9 & 10 \\ 1 & 2 & 3 & 4 & 5 & 6 & 7 & 8 & 9 & 10 \\ 1 & 2 & 3 & 4 & 5 & 6 & 7 & 8 & 9 & 10\end{array}$

3. Estructura, organización y desarrollo del artículo

4. Interés y originalidad en el tratamiento del tema

5. Referencias bibliográficas: relevancia y actualidad

6. Antecedentes: revisión de la literatura

7. Metodología del trabajo

8. Resultados y conclusiones: aportación al conocimiento científico

9. Aspectos formales: redacción, claridad, rigor conceptual

10. Valoración global

\section{VALORACIÓN FINAL}

A. Publicable tal como está (corrigiendo algún aspecto de forma señalado)...

B. Publicable, con las correcciones y mejoras señaladas.

C. No publicable, por razones señaladas

Modificaciones, correcciones, sugerencias (en caso de A o B)

Fundamentos de la resolución (en caso de C)

Anotaciones para el director, de uso exclusivo

Universidad de Salamanca. Facultad de Educación.

Departamento de Teoría e Historia de la Educación.

C/ Paseo de Canalejas, 169. 37008 Salamanca.

Teléfono: 923294 630, extensión 3391, agd@usal.es 



\section{ARTICLE INFORMATION}

Title:

Date of reception:

Date returned:

Reviewer:
Date sent for review:

Date of final acceptance:

\section{ASSESSMENT CRITERIA}

1. The title is in line with the content of the work

2. Structure and contents of abstract and key words

3. Structure, organization and development of the article

4. Interest of and originality in the treatment of the topic

5. Bibliographical references: relevance and topicality

6. Background: literature review

7. Work methodology

8. Results and conclusion: contribution to scientific knowledge

9. Formal aspects: writing, clarity, conceptual rigour

FINAL ASSESSMENT

A. Publishable as is (with correction of formal aspects indicated).

B. Publishable, with the corrections and improvements indicated

C. Not publishable.

Modifications, corrections, suggestions (If A B)

Basis of decision (If C)

Notes exclusively for the editor

Universidad de Salamanca. Facultad de Educación.

Departamento de Teoría e Historia de la Educación.

C/ Paseo de Canalejas, 169. 37008 Salamanca.

Telephone: 923294 630, extension 3391, agd@usal.es 

ISSN 1130-3743

\section{DONNÉES DE L'ARTICLE}

Titre:

Date de réception:

Date de renvoi:

Referee:
Date d'envoi à évaluation:

Date d'acceptation définitive:

\section{CRITÈres D'ÉVALUATION}

1. Adéquation du titre aux contenus du travail

2. Structure et contenu du résumé et mots clés

3. Structure, organisation et développement de l'article

4. Intérêt et originalité du sujet

5. Références bibliographiques: importance et nouveauté

6. Précédents: révision de la littérature

7. Méthodologie du travail

$\begin{array}{llllllllll}1 & 2 & 3 & 4 & 5 & 6 & 7 & 8 & 9 & 10\end{array}$

$\begin{array}{llllllllll}1 & 2 & 3 & 4 & 5 & 6 & 7 & 8 & 9 & 10\end{array}$

$\begin{array}{llllllllll}1 & 2 & 3 & 4 & 5 & 6 & 7 & 8 & 9 & 10\end{array}$

$\begin{array}{llllllllll}1 & 2 & 3 & 4 & 5 & 6 & 7 & 8 & 9 & 10\end{array}$

$\begin{array}{llllllllll}1 & 2 & 3 & 4 & 5 & 6 & 7 & 8 & 9 & 10\end{array}$

$\begin{array}{llllllllll}1 & 2 & 3 & 4 & 5 & 6 & 7 & 8 & 9 & 10\end{array}$

$\begin{array}{llllllllll}1 & 2 & 3 & 4 & 5 & 6 & 7 & 8 & 9 & 10\end{array}$

8. Résultats et conclusions: apports à la connaissance scientifique

$\begin{array}{llllllllll}1 & 2 & 3 & 4 & 5 & 6 & 7 & 8 & 9 & 10\end{array}$

$\begin{array}{llllllllll}1 & 2 & 3 & 4 & 5 & 6 & 7 & 8 & 9 & 10\end{array}$

$\begin{array}{llllllllll}1 & 2 & 3 & 4 & 5 & 6 & 7 & 8 & 9 & 10\end{array}$

\section{QUALIFICATION GLOBALE}

A. Publiable tel qu'il est (en corrigeant quelques aspects formels signalés).....

B. Publiable avec les corrections et améliorations signalées

C. Non publiable

Modifications, corrections, suggestions (si A ou B)

Base de la décision (C)

Observations pour le directeur, d'utilisation exclusive

Universidad de Salamanca. Facultad de Educación.

Departamento de Teoría e Historia de la Educación.

C/ Paseo de Canalejas, 169. 37008 Salamanca.

Telephone: 923294 630, extension 3391, agd@usal.es 
